\title{
A minute-continuous-wave-stabilized picosecond supercontinuum source for ultrafast serial time-encoded amplified microscopy (STEAM)
}

\author{
Chi Zhang, Yi Qiu, Jianbing Xu, Kenneth K. Y. Wong, and Kevin K. Tsia* \\ Department of Electrical and Electronic Engineering, The University of Hong Kong, Pokfulam Road, Hong Kong \\ tsia@hku.hk
}

\begin{abstract}
A stabilized picosecond supercontinuum source, by a minute continuous-wave trigger, is utilized to improve the ultrafast imaging quality of serial time-encoded amplified microscopy (STEAM) with a frame rate of $4.9 \mathrm{MHz}$.

OCIS codes: (170.0110) Imaging systems; (180.0180) Microscopy; (320.6629) Supercontinuum generation.
\end{abstract}

Stable supercontinuum (SC) source is of great importance for a diverse range of applications in, for instance, frequency metrology, spectroscopy, hyperspectral imaging. Nevertheless, achieving a robust control of the stability of SC remains a critical challenge in many areas especially for those which require real-time, ultrafast and singleshot spectral measurements [1,2]. One example is serial time-encoded amplified microscopy (STEAM), which was recently demonstrated as a real-time ultrafast and sensitive optical imaging technique [2,3]. Imaging in STEAM is achieved by mapping the spectrally-encoded image shots into individual optically-amplified temporal pulses. Thus, a broadband pulse source (e.g. SC) with a high-degree of shot-to-shot stability represents the key enabling factor in STEAM. Although it has been known that SC stability can be improved by using femtosecond pulses, such ultrashort pulses require dedicated dispersion control and are often sensitive to perturbations. Longer-pulse SC (e.g. picoseconds or nanoseconds) are hence more practical and indeed have widely been utilized in a myriad of biophotonics applications [4]. However, long-pulse SC suffers from significant pulse-to-pulse fluctuation which primarily stems from modulation instability (MI) - a nonlinear process initiates spontaneously from noise [5]. Such noisy behavior explains why the long-pulse SC sources have been precluded in all the previously reported STEAM systems, which instead employed the femtosecond SC source [2].

Various schemes have been proposed and implemented to control the SC instability, such as feedback mechanism [6], dispersion engineering [7], pulse seeding approach, or THz intensity modulation of the input pulse [8]. In contrast to the prior works, we here demonstrate a simple triggering mechanism to enhance and stabilize picosecond SC generation by introducing a minute continuous-wave (CW) light within the MI gain spectral regions [9]. This CW-triggering technique does not require phase-locking with the pump and precise time-delay tuning (as opposed to the pulse-seeding scheme [10]). Hence, it represents a handy approach to achieve active control of SC stability and to help extend the applicability of SC in STEAM from the femtosecond to the picosecond or even nanosecond regimes. In particular, we demonstrate single-shot STEAM imaging at a frame rate of $4.9 \mathrm{MHz}$ using the CW-triggered picosecond SC source. More importantly, such CW-stabilized SC can greatly reduce the shot-toshot STEAM image fluctuation.

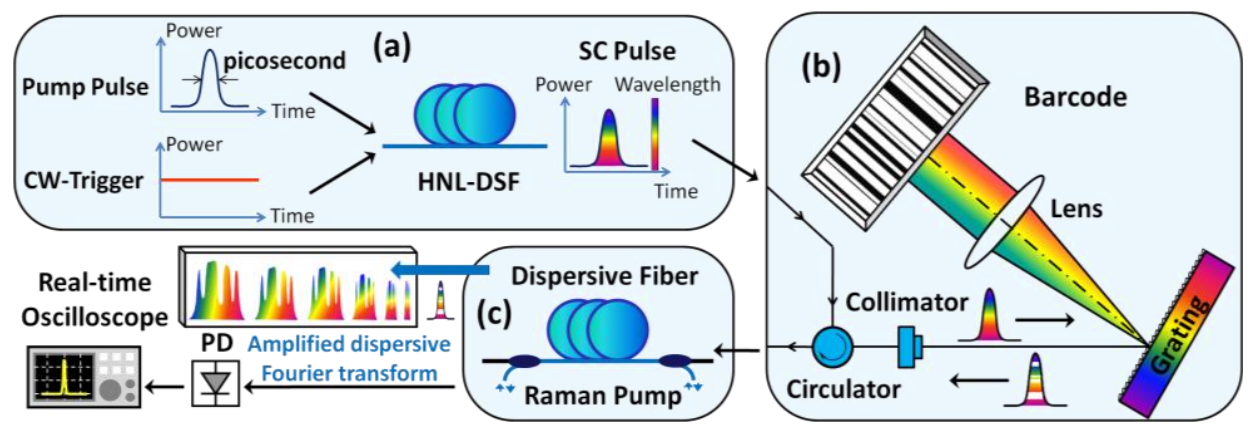

Fig. 1. Schematic of the STEAM system based on the CW-stabilized picosecond SC source. (a) CWtriggering mechanism for SC generation; (b) Space-wavelength mapping: a diffraction grating is used to encode the spaial information into the spectrum; (c) Wavelength-time mapping: A Raman-amplified dispersive fiber maps the spectrally encoded image of a sample into a temporal waveform, which is then captured by the photodetector and digitized by the real-time oscilloscope. 
The experimental setup for generating stabilized SC source based on the CW-triggering mechanism is illustrated in Fig. 1 (a). Intense pump pulses delivered by a picosecond mode-locked fiber laser (pulse width 5.8 ps, peak power $22 \mathrm{~W}$, wavelength $1554.5 \mathrm{~nm}$ ), as well as a $\sim 80 \mu \mathrm{W} \mathrm{CW}$ source, are coupled together into a 50 m highly-nonlinear dispersion-shifted fiber (HNL-DSF) (zero-dispersion wavelength $1554 \mathrm{~nm}$, nonlinear coefficient: $14 \mathrm{~W}^{-1} \mathrm{~km}^{-1}$ ). As shown in Fig. 2(a), the SC spectrum is greatly enhanced when the CW wavelength approaches the MI gain sidebands (1500-1510 $\mathrm{nm}$ and 1610-1620 nm), where it experiences large MI gain. Notably, when the CW-trigger is at $1615 \mathrm{~nm}$, the enhanced SC spectrum spans from $1400 \mathrm{~nm}$ to $1700 \mathrm{~nm}$, which is much wider than the untriggered spectrum, and the spectral power is increasing by $\sim 20 \mathrm{~dB}$ on both the redshifted and blueshifted sides of the SC spectrum. Fig. 2 (b) shows the amplitude histogram of 781 filtered SC pulses (1620-1650 nm). In untriggered case, it shows a clear long-tail distribution, resembling the extreme-value statistics [11]. In contrast, when the CW-trigger is added, it alters the SC amplitude statistics to an almost-Gaussian distribution and the CWtriggered SC shows a $\sim 50 \%$ reduction in the standard deviation. Such improvement in pulse-to-pulse stability is also readily evident from the real-time pulse traces in both untriggered and CW-triggered cases (insets of Fig. 2(b)). Such CW-stabilized picosecond SC pulses are favorable for high-speed and single-shot STEAM imaging.
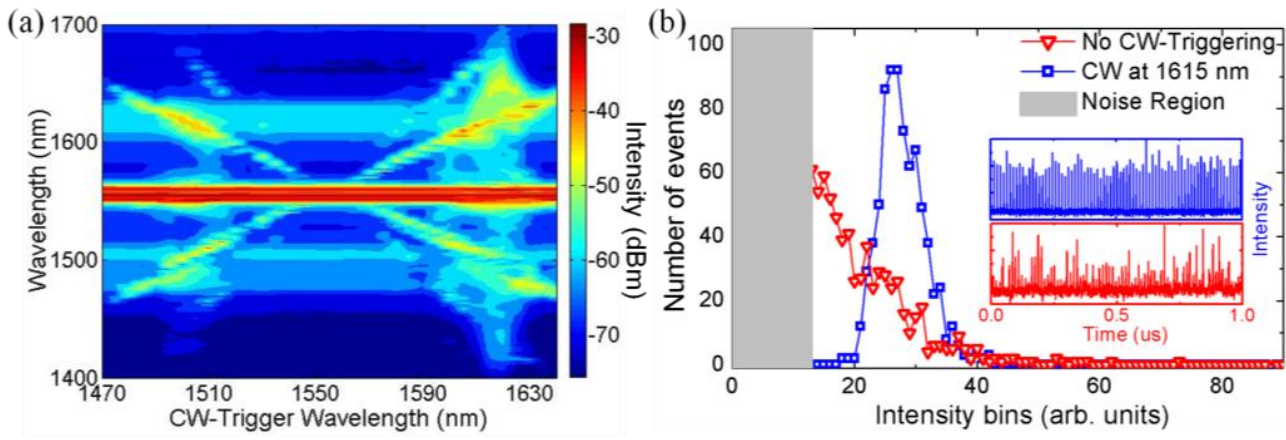

Fig. 2. CW-triggered SC source. (a) The measured SC spectra as a function of the CW-trigger wavelength; (b) Filtered pulse-to-pulse amplitude histograms of the untriggered SC (triangles) and the CW-triggered SC (squares), with similar average power level. The shaded region represents the noise floor of the measurement. The insets show the real-time pulse traces of (lower inset) the untriggered and (upper inset) the CW-triggered SC.

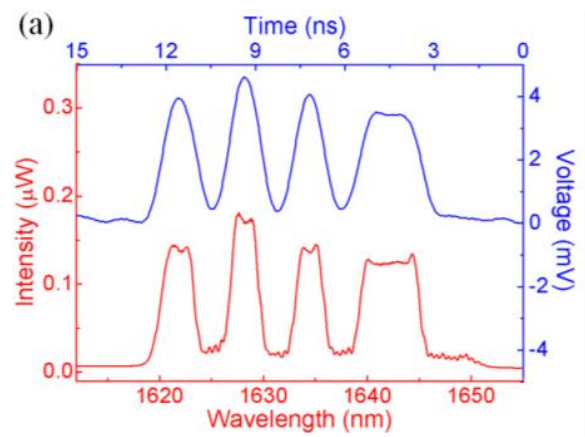

(b)

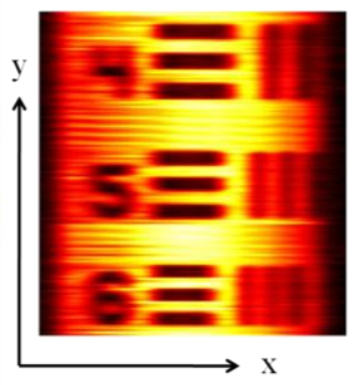

(c)

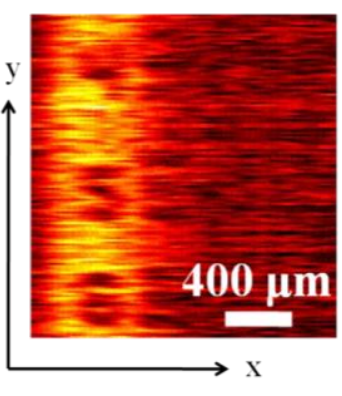

Fig. 3. (a) Comparison between the temporal waveform (single shot) captured by the oscilloscope (blue top) and the spectrum measured by a conventional optical spectrum analyzer (red bottom). The consistency between them validates the frequency-to-time mapping operation. Images of a resolution target (USAF 1951) captured by STEAM using (b) the CW-triggered SC and (c) the untriggered SC.

Here, the key feature of STEAM is the mapping of an image into a serial time-domain waveform by a two-step approach: (i) space-wavelength mapping - the spatial information of a sample is first encoded onto the spectrum of the CW-stabilized SC pulse by using a spatial disperser (e.g. diffraction grating) (Fig. 1(b)). (ii) wavelength-time mapping - A dispersive element (e.g. a dispersive fiber) is then used to perform a process called amplified dispersive Fourier transform (ADFT) which maps the spectrum of an optical pulse into a temporal waveform using group-velocity dispersion (GVD) [1] (Fig. 1(c)). The image is detected with a single-pixel photodiode and captured with a real-time oscilloscope $(4 \mathrm{GHz}, 20 \mathrm{GS} / \mathrm{s})$. We employ a diffraction grating (600 lines/mm) together with an imaging lens (focal length of $50 \mathrm{~mm}$ ) to perform the space-wavelength mapping. Then, we use a dispersion compensation fiber (DCF) (a dispersion of $-867 \mathrm{ps} / \mathrm{nm}$ ) to temporally disperses and maps the spectrally-encoded information of the SC pulse into a temporal waveform. Within the same DCF, we also perform Raman amplification 
(13-dB optical gain) to overcome the loss inherent in the dispersive medium [1]. Fig. 3(a) illustrates the consistency between the spectral information and the temporal waveform in STEAM and hence verifies the space-to-time mapping operation of STEAM.

Fig. 3(b) and (c) shows the impact of the CW-stabilized SC on the image quality of STEAM. As a proof-ofconcept demonstration, the present STEAM system operates in the single-shot line scan mode (along the $\mathrm{x}$-direction) at a rate of $4.9 \mathrm{MHz}$ (effectively determined by the repetition rate of the source). The two-dimensional (2D) images are reconstructed by translating the sample in the orthogonal direction (along the y-direction). We note that such line-scan STEAM configuration naturally finds a compelling application in high-speed and sensitive imaging flowcytometry [12]. Fig. 3(b) shows the STEAM image of the USAF-1951 standard resolution target taken by the CWtriggered SC source. Clearly, the CW-triggered SC results in a better STEAM image quality when it is compared with the image taken by the untriggered SC (Fig. 3(c)), which shows a high level of image noise. Similar comparison is also made for lens paper imaging (Fig. 4). For the case of STEAM using the untriggered SC, the fiber structures in the lens paper are hardly visualized and the image suffers serious noise contamination (Fig. 4(a)). In contrast, the lens paper STEAM image taken by the CW-triggered SC (Fig. 4(b)) shows a greatly improved image contrast - demonstrating the impact of the CW-stabilized picoseconds SC source on the imaging performance of STEAM.

Notably, the fact that STEAM is a confocal imaging technique [13] can be understood by noticing the out-offocus features in the STEAM image (Fig. 4(b)) are missing when compared with the conventional bright-field microscope image (Fig. 4 (c)). We also note that the spatial resolution in the present STEAM system can easily be further improved by increasing the numerical aperture of the lens, GVD and the bandwidth oscilloscope [3].

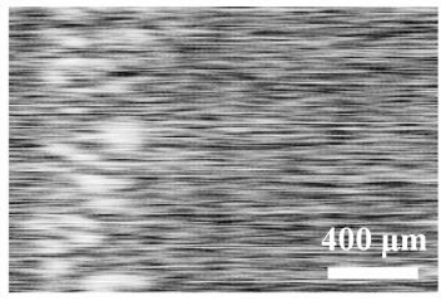

(a)

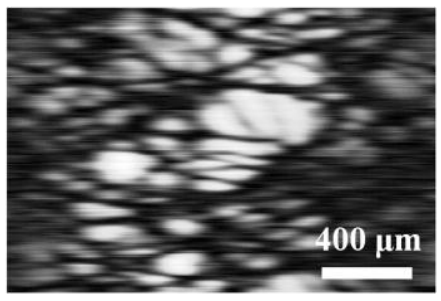

(b)

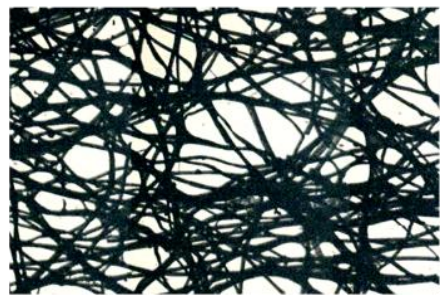

(c)

Fig. 4. Images of a lens paper captured by STEAM using (a) untriggered SC and (b) CW-triggered SC. (c) A bright field image of the same area (as in (a) and (b)).

In conclusion, we have experimentally demonstrated a simple active triggering scheme to enhance and stabilize the long-pulse (picosecond) SC without resorting to the complex techniques such as precise time delay tuning, phase-locking, dedicated feedback control. More importantly, we show that this handy CW-stabilizing approach enables, for the first time, STEAM operation in the long SC pulse (picosecond) regime - extending the applicability of SC in STEAM from the femtosecond to the picosecond or even nanosecond regimes. Specifically, we demonstrated that the image quality of ultrafast single-shot STEAM (at a rate of 4.9MHz) can be greatly enhanced by using such CW-triggered SC By further improving the image resolution, we expect that STEAM could find a wide range of appealing applications in which real-time, high-speed and high-throughput imaging is essential, e.g. imaging flow cytometry.

The work in this paper is partially supported by grants from the Research Grants Council of the Hong Kong Special Administrative Region, China (Project No. HKU 7179/08E, HKU 7183/09E and HKU 717510E).

[1] K. Goda, D. R. Solli, K. K. Tsia, and B. Jalali, Phys. Rev. A 80, 043821 (2009).

[2] K. Goda, K. K. Tsia, and B. Jalali, Nature 458, 1145-1149 (2009).

[3] K. K. Tsia, K. Goda, D. Capewell, and B. Jalali, Opt. Express 18, 10016-10028 (2010).

[4] J. M. Dudley and J. R. Taylor, Supercontinuum generation in optical fibers (Cambridge University Press, 2010).

[5] G. Genty, S.Coen, and J. M. Dudley, J. Opt. Soc. Am. B 24, 1771-1785 (2007).

[6] P. M. Moselund, M. H. Frosz, C. L. Thomsen, and O. Bang, Opt. Express 16, 11954-11968 (2008).

[7] J. N. Kutz, C. Lyngå, and B. J. Eggleton, Opt. Express 13, 3989-3998 (2005).

[8] G. Gently, J. Dudley, and B. Eggleton, Appl. Phys. B: Lasers Opt. 94, 187-194 (2009).

[9] K. K. Y. Cheung, C. Zhang, Y. Zhou, K. K. Y. Wong, and K. K. Tsia, Opt. Lett. 36, 160-162 (2011).

[10] D. R. Solli, C. Ropers, P. Koonath, and B. Jalali, Phys. Rev. Lett. 101, 233902 (2008).

[11] D. R. Solli, C. Ropers, P. Koonath, and B. Jalali, Nature 450, 1054-1057 (2007).

[12] J. V. Watson, Introduction to flow cytometry (Cambridge Univ. Press, 2004).

[13] J. B. Pawley, Handbook of biological confocal microscopy (Springer, 2006). 